\title{
Toward standardized procedures for recording and describing 3-D shoulder movements
}

\author{
H. E. J. VEEGER \\ Vrije Universiteit, Amsterdam, The Netherlands \\ and Technische Universiteit Delft, The Netherlands \\ and \\ F. C. T. VAN DER HELM, E. K. J. CHADWICK, and D. MAGERMANS \\ Technische Universiteit Delft, The Netherlands
}

\begin{abstract}
Movements of the human upper extremity are difficult to measure, due to the specific structure of the limb. All arm movements are a combination of movements of the humerus relative to a moveable base: the scapula. Measurement of movements is, however, strongly hampered by the fact that the scapular movement is difficult to track when skin-based landmarks are used. A second complicating factor is the fact that a skin-based proximal landmark on the humerus is difficult to define, which directly affects attempts to describe accurately the movements of that segment. To solve these problems, special measurement procedures have been developed, which include the identification of landmarks for the scapula and the humerus and recording procedures for segment movements or the estimation of landmarks. Also, a definition of the three-dimensional description of motion is presented. These measurement procedures have now been applied in several experiments and have been proven to be successful.
\end{abstract}

Although the shoulder mechanism is often loosely referred to as a single ball-and-socketjoint between the trunk and the arm, the shoulder mechanism consists of three segments-namely, the humerus, the clavicle, and the scapula - that move relative to the thorax (Figure 1). The shoulder mechanism comprises three synovial jointsnamely, the sternoclavicular joint, the acromioclavicular joint, and the glenohumeral joint. The medial border of the scapula is pressed against the thorax by the combined action of the $\mathrm{m}$. serratus anterior and the m. rhomboideus. This connection, the so-called scapulothoracicgliding plane, turns the shoulder girdle into a closed-chain mechanism.

The complex motion constraints of the shoulder girdle result in a three-dimensional (3-D) motion pattern. As a consequence, motion studies that include the shoulder should be performed in three dimensions, and 3-D motion recordings and descriptions are necessary. In addition, the fact that scapular motions contribute to overall arm motions makes inclusion of scapular motions in recordings (and descriptions) necessary. If the shoulder is described as a generic thoracohumeral joint, its rotation center lies well outside the glenohumeral joint. Doorenbosch, Mourits, Veeger, Harlaar, and van der Helm (2001), in a study on the functional rotation axis of the shoulder complex, esti-

Correspondence concerning this article should be addressed to H. E. J. Veeger, Institute for Fundamental and Clinical Human Movement Sciences, Department of Human Movement Sciences, Vrije Universiteit, v.d. Boechorststraat 9, 1081 BT Amsterdam, The Netherlands (e-mail: h_e_j_veeger@fbw.vu.nl). mated that for movements in the frontal plane, this axis was located about $13 \mathrm{~cm}$ medially relative to the acromion.

Three-dimensional scapular, and clavicular, motions have been studied with invasive methods, such as 3-D Xray combined with implanted markers (Högfors, Peterson, Sigholm, \& Herberts, 1991) or electromagnetic systems combined with bone pins (Karduna, McClure, \& Michener, 2000; Lu, Li, Kuo, Chang, \& Hsu, 2002). The invasive character of these methods is, however, a serious drawback for their use as methods in diagnostic or evaluative research.

To overcome the difficulties related to invasive methods, researchers have used a palpation technique in which bony landmarks were retrieved and subsequently digitized, using a palpator (van der Helm \& Pronk, 1995; Veeger, van der Helm, \& Rozendal, 1993), electromagnetic devices such as 3-space or flock-of-birds (Johnson, Stuart, \& Mitchell, 1993; Meskers, van der Helm, Rozendaal, \& Rozing, 1998), or opto-electronic devices (Hebert, Moffet, McFadyen, \& St-Vincent, 2000). The palpation method is, however, static: Only positions can be measured, not motions. Karduna, McClure, Michener, and Sennett (2001) performed a validation study to compare two noninvasive methods, using an electromagnetic sensor with results from bone pin measurements. The noninvasive techniques involved fixing a sensor directly to the acromion and mounting a sensor on the acromion with the use of an adjustable rig that was fitted over the spine of the scapula and the acromion. The authors (Karduna et al., 2001) concluded that the average motion pattern of the noninvasive techniques was similar to that of the invasive technique, especially below $120^{\circ}$ of arm elevation. 


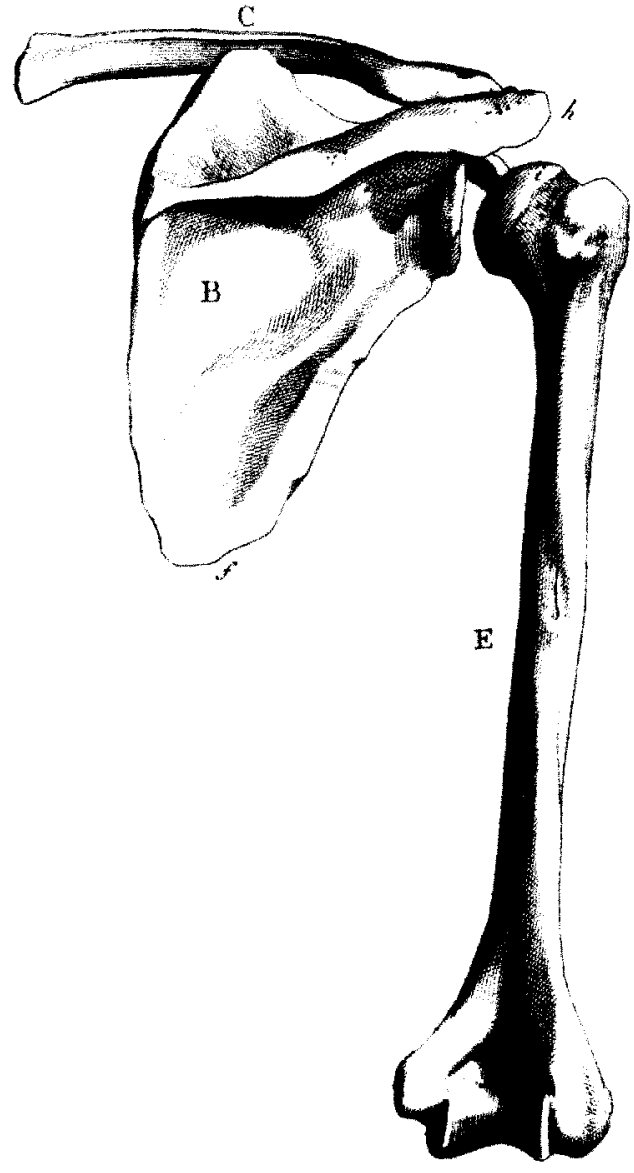

Figure 1. Rear view of the bony parts of the human shoulder (from Giuseppe del Medico, Anatomia per Uso dei Pittori e Scultori, 1811). B, shoulder blade or scapula; C, clavicle; E, upper arm or humerus; $h$, acromion; $f$, inferior angle of the scapula.

Although some attempts may have been made to use optic recording systems for the measurement of armshoulder movements with markers on the skin (Leroux, Micallef, Bonnel, \& Blotman, 1992; Sharp, Suur, \& Woledge, 2001; Vaughan, Rosenbaum, Harp, Loukopoulos, \& Engelbrecht, 1998), these recordings do not track scapular movements adequately, due to the large displacements that occur between bone and skin during clavicular and scapular motions.

Accurate registration of the position or orientation of a segment in three dimensions requires at least three identification points on that segment, so that a local coordinate system can be constructed. Usually, standard anatomical landmarks are used for these points. To optimize the accuracy of the analyses, it is necessary that these anatomical landmarks be chosen so that they can be used for the reliable construction of a local coordinate system. This requires a sufficient spread between those markers. For the thorax and the scapula (Figure 2), this requirement does not lead to difficulties. However, for the humerus, the definition of an easily identifiable proximal landmark is, in fact, impossible when standard palpation techniques are used. In addition, the accurate estimation of axial rotations of the humerus is hampered by the small distance between the available distal anatomical landmarks on both epicondyles. For the clavicle, it is virtually impossible to discern three noncolinear landmarks.

This article describes a procedure for the in vivo noninvasive measurement of arm-shoulder motion that includes the scapula. The description focuses on the procedures for the measurement of the kinematics of the scapula and the humerus. These comprise the choice of landmarks for the definition of a local coordinate system and the methods for obtaining scapular motion data, with special attention to the relationship between the two.

\section{Bony Landmarks and Local Coordinate Systems}

Local coordinate systems are necessary to be able to describe the position and orientation of a segment relative to the outside world or to another segment (see Berme, Capozzo, \& Meglan, 1990). Since the shapes of bones and segments are different between individuals, it must be acknowledged that exactly identical reference frames for comparing motions do not exist and that standardized definitions defined on anatomical landmarks are the best possible alternative. Bony landmarks are defined as easily retrievable reference points on a bone and are preferred above bony ridges, such as the scapular spine or the humeral shaft. Bony landmarks are used to define local coordinate systems of segments (Table 1). For comparison of motions between studies, it is essential that the same bony landmarks be used, since these determine the local coordinate system that is being used.

The International Shoulder Group (ISG) has proposed to the International Society of Biomechanics a series of standard definitions for use as local coordinate systems (van der Helm et al., in press). These definitions are based

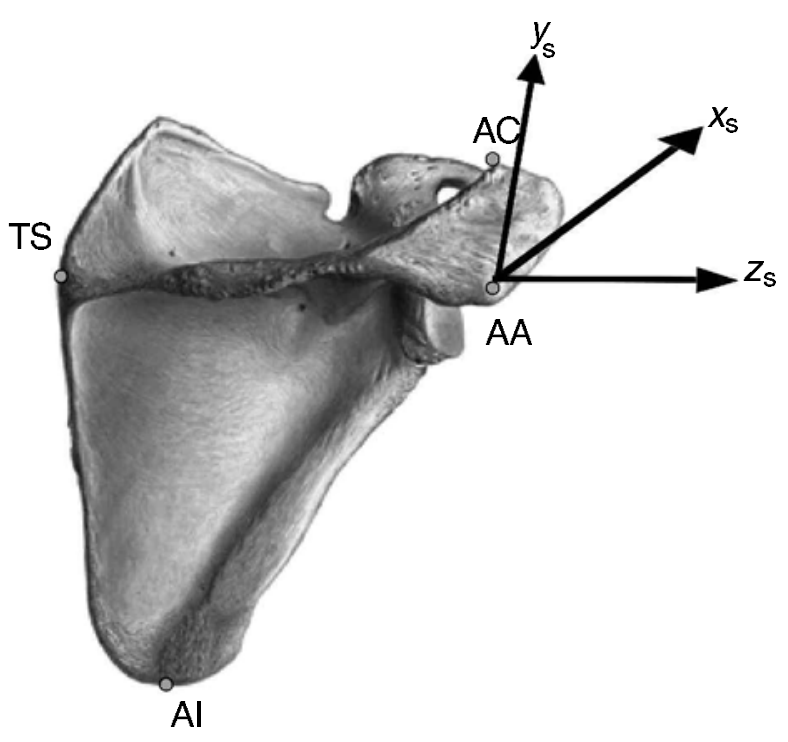

Figure 2. Bony landmarks and local coordinate system of the scapula. 
Table 1

Description of Bony Landmarks

\begin{tabular}{lcl}
\hline Segment & Abbreviation & Description \\
\hline Thorax & IJ & deepest point of incisura jugularis (suprasternal notch) \\
& PX & processus xiphoideus, most caudal point on sternum \\
& C7 & processus spinosus of 7th cervical vertebra \\
& T8 & processus spinosus of 8th thoracic vertebra \\
Clavicle & SC & most ventral point on sternoclavicular joint \\
& AC & most dorsal point on acromioclavicular joint (shared with scapula) \\
Scapula & AC & most dorsal point on acromioclavicular joint (shared with clavicula) \\
& TS & trigonum spinae scapulae, midpoint of triangular surface on medial \\
& bi & border of the scapula in line with the scapular spine \\
& AA & angulus inferior, most caudal point of scapula \\
& PC & most ventral point of processus coracoideus \\
Humerus & glenohumeral rotation center, estimated by regression or motion \\
& GH & recordings \\
& EM & most caudal point on medial epicondyle \\
& EL & most caudal point on lateral epicondyle \\
& US & most caudal-medial point on the ulnar styloid \\
& RS & most caudal-lateral point on the radial styloid \\
\hline
\end{tabular}

on the following principles: The global coordinate system and the local coordinate systems of the bones are aligned as much as possible in the initial (resting) position; rotations about intuitively recognizable axes are more readily interpretable; and gimbal lock orientations anywhere in the range of motion must be avoided. The basis of the definitions is formed by the definition of the thorax local coordinate system. Although "shoulder" movements should, in principle, be described as the rotations of the humerus relative to its proximal segment, the scapula, these movements are often described relative to the thorax. These thoracohumeral descriptions are used especially in more clinically oriented function evaluations of the upper extremity (Anglin \& Wyss, 2000). The definition of the thorax local coordinate system is as follows.

Thorax Coordinate System: $x_{\mathrm{t}} y_{\mathrm{t}} z_{\mathrm{t}}$

$o_{\mathrm{t}}$ : The origin coincident with the incisura jugularis (IJ; see Table 1 for a description of bony landmarks).

$y_{\mathrm{t}}$ : The line connecting the midpoint between PX and T8 and the midpoint between the IJ and C7, pointing upward.

$z_{\mathrm{t}}$ : The line perpendicularto the plane formed by the IJ, C7, and the midpoint between PX and T8, pointing to the right.

$x_{\mathrm{t}}$ : The common line perpendicularto the $x_{\mathrm{t}}$ - and the $y_{\mathrm{t}}$-axis, pointing forward.

The anatomical landmarks that are needed for definition of the local coordinate system of the scapula are easy to determine. Standard anatomical landmarks are the inferior angle of the scapula, the trigonum spinae scapulae, and the angulus acromialis (Table 1, Figure 2). Although previous studies used and promoted the use of a point on the acromiclavicular joint (Karduna et al., 2001; Veeger et al., 1993), more recently, the acromial angle was proposed as the third landmark (de Groot, 1997), mainly to reduce the chances that Gimbal lock would occur. The scapula coordinate system is then defined as follows.

Scapula Coordinate System: $x_{\mathrm{s}} y_{\mathrm{s}} z_{\mathrm{s}}$

$o_{\mathrm{s}}$ : The origin coincident with AA. $z_{\mathrm{s}}$ : The line connecting AA and TS, pointing to AA.

$x_{\mathrm{s}}$ : The line perpendicular to the plane formed by AI, AA, and $x_{\mathrm{s}}$, pointing forward.

$y_{\mathrm{s}}$ : The common line perpendicularto the $x_{\mathrm{s}}$ - and the $z_{\mathrm{s}}$-axis.

\section{Recording Procedures for Motions of the Scapula}

Of course, valid recording of motions including the shoulder mechanism should comprise scapular motions, either directly or indirectly. Since standard optical methods are not feasible, there are essentially three options by which to overcome this problem.

1. Quasi-static measurements, during which a particular task is divided into different steps, or snapshots, in which the orientation of the trunk, the scapula, and the arm are measured. These measurements can be done by palpation with a scapula locator, a triangular device with adjustable pins that can be placed over the anatomical landmarks of the scapula. A sensor on the device is then used for the recording of the positions of those landmarks (Johnson et al., 1993). Alternatively, a marker tree can be used in combination with a scapula locator.

2. When dynamic measurements are involved, the orientation of the scapula can be estimated from the measurement of trunk motion and arm motion, using regression equations. These regression equations (de Groot \& Brand, 2001; Pascoal, van der Helm, Correia, \& Carita, 2000; Veeger et al., 1993) are based on the existence of a relatively stable relationship (Karduna, Ebaugh, Michener, \& McClure, 2002) between scapular movements and the elevation of the humerus. A disadvantage of this method, however, is the fact that for an optimal definition of the motions of the humerus, the center of the humeral head is the most suitable landmark. For the definition of this landmark, the orientation of the scapula is required, which can be derived only with this method, not directly measured.

3. Direct measurement of scapular kinematics using an electromagnetic sensor (or a marker tree) on the acromion, in combination with calibration measurements to define 
its relationship with anatomical landmarks (Karduna et al., 2001). Although this method has been proven to lead to good results at lower elevation angles, its accuracy during higher elevation is still uncertain. The essential step in this procedure is the calibration step, which is based on a rigid-body assumption. When the positions of the anatomical landmarks relative to the sensor are known, the positions of those landmarks can be reconstructed from sensor data at any given sample.

\section{Standardized Motion Descriptions of the Scapula}

The rotations of the scapula can be expressed as different combinations of Euler angles, which are dependent on the order of decomposition of the orientation matrices. The other way around, Euler angles derive their meaning from the rotation order that is being used: Different sequences of rotations can lead to completely different orientations. In agreement with Karduna et al. (2000), the protraction/ retraction-lateral/medial-rotation-anterior/posterior-tilt order (Figure 3 ) is the preferred combination of rotations, since it is both stable and as close as possible to the twodimensional movement descriptions in vogue. This order is also in agreement with the proposed guidelines by the ISG (van der Helm et al., in press).

\section{Recording Procedures for Motions of the Humerus}

It is quite difficult to define three anatomical landmarks for the humerus that are spread over the segment adequately. On the distal end of the humerus, the medial and lateral epicondyles can easily be defined, but it is virtually impossible to define an easily identifiable proximal landmark. This is of course due to the fact that the humeral head is completely covered by muscles (the rotator cuff muscles, as well as the deltoid muscle). The best solution for this problem is to define the glenohumeral rotation center (more or less equal to the center of the humeral head) as the local landmark. The definition of the local coordinate system is then the following.

Humerus (1 st degree) Coordinate System: $x_{\mathrm{h} 1} y_{\mathrm{h} 1} z_{\mathrm{h} 1}$

$o_{\mathrm{h} 1}$ : The origin coincident with $\mathrm{GH}$.

$y_{\mathrm{h} 1}$ : The line connecting $\mathrm{GH}$ and the midpoint of EL and $\mathrm{EM}$, pointing to $\mathrm{GH}$.

$x_{\mathrm{h} 1}$ : The line perpendicular to the plane formed by EL, $\mathrm{EM}$, and $y_{\mathrm{h} 1}$, pointing forward.

$z_{\mathrm{h} 1}$ : The common line perpendicularto the $y_{\mathrm{h} 1^{-}}$and the $z_{\mathrm{h} 1^{-}}$ axes.

The glenohumeral rotation center can be defined either on the basis of kinematic recordings in a calibration procedure or from regression equations that describe the position of the center of the humeral head relative to four anatomical landmarks on the scapula.

1. If the glenohumeral rotation center is estimated on the basis of kinematic recordings, information is needed on the movements of the humerus relative to the scapula. These data can be obtained from measurements in which both the scapula and the humerus are recorded. For the scapula, this can be done by placing a marker on the acromion (see above; Karduna et al., 2001). Alternatively, when a scapula locator is being used, this locator should be kept fixed on the scapula. If arm elevation angles are low, this procedure appears to be sufficiently accurate (Stokdijk, Nagels, \& Rozing, 2000). From the relative rotations and displacements of the humerus to the scapula, a rotation center can be estimated by fitting a sphere on the movements of the humerus (Karduna et al., 2000) or by the calculation of the intersection of instantaneous helical axes (Stokdijk et al., 2000; Veeger, 2000; Veeger, Yu, An, \& Rozendal, 1997). The latter method is, in theory, the most correct method, since it takes axial rotations into

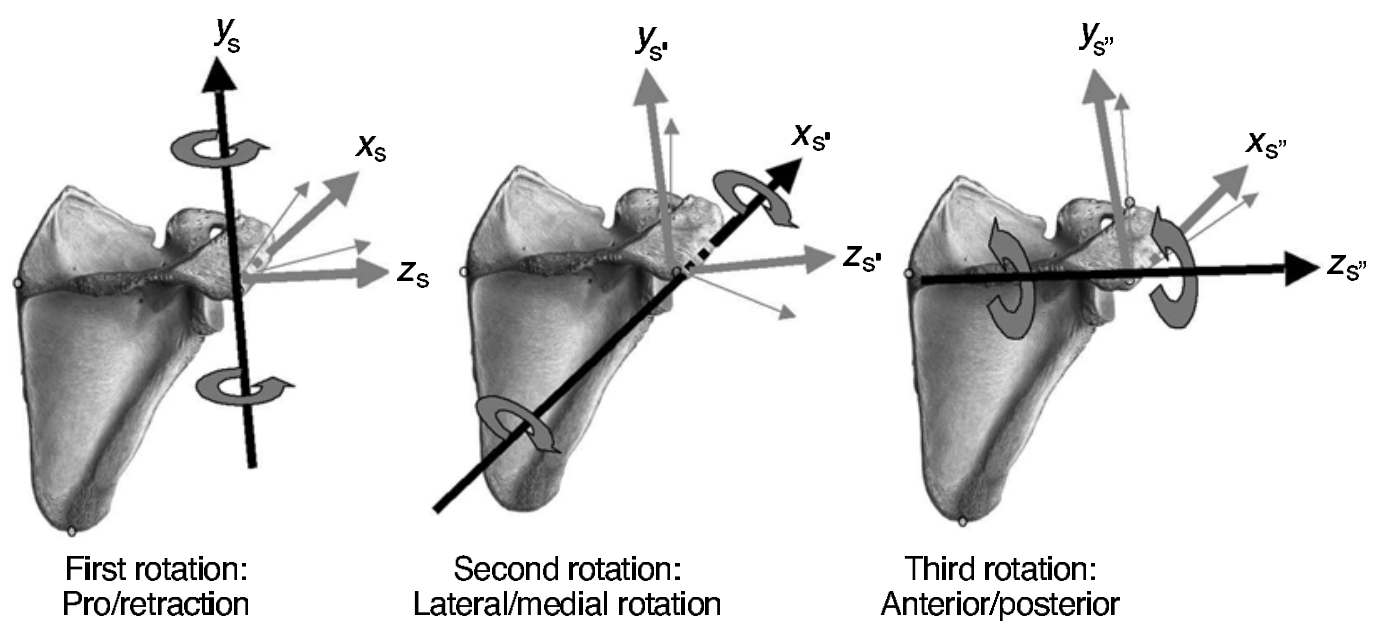

Figure 3. Rotation order for the scapula. The scapula is drawn from the rear, and the $x_{\mathrm{s}}$-axis is therefore pointing forward. The single quotes or double quotes indicate that the axes have been rotated, due to previous rotations. 
account. According to Stokdijk et al. the instantaneous helical axis method is the preferred method, due to the fact that the method can also be applied to hinge joints.

2 . The center of the humeral head can also be obtained from regression equations based on the measurement of anatomical landmarks on the scapula. Meskers et al. (1998) showed that a very close relationship exists between the shape of the scapula and the factors that determine the position of the center of the humeral head: the orientation of the glenoid and the size of the humeral head. To obtain the coordinates for the humerus rotation center, information is needed on AC or AA, TS, AI, and the coracoid process (see Table 1).

Although the two distal landmarks on the humerusnamely, EL and EM (Table 1) - are easily definable, the distance between those landmarks is rather small. As a consequence, the effect of measurement errors can become troublesome. To overcome this, an alternative system has been proposed that uses the plane formed by the upper arm and the forearm (when the elbow is flexed $90^{\circ}$ ) for estimation of the $z_{\mathrm{h}}$ local coordinate axis (2nd degree definition of the humerus; see the Appendix). The two definitions of the coordinate systems will not exactly agree, mainly because the long axis of the forearm is not necessary perpendicular to the line EM-EL, dependent on the carrying angle of the elbow and the orientation of the flexion-extension axis relative to the line EM-EL (Veeger et al., 1997).

\section{Standardized Motion Descriptions of the Humerus}

Thoracohumeral motions have often been expressed according to the globographic convention (Anglin \& Wyss,
2000; Pearl et al., 1992). According to this description, the positions of the humerus are described as rotations of the elevation (or longitude on a globe)-elevation-angle (or latitude on a globe)-axial-rotation plane (Figure 4). The same order, in fact, applies to glenohumeral rotation: the rotation of the humerus relative to the scapula (An, Browne, Korinek, Tanaka, \& Morrey, 1991; Vermeulen et al., 2002). It should, however, be kept in mind that although the order of rotation is the same for thoracohumeral motion and glenohumeral motion, the rotation angles are not: Outward rotation of the scapula accounts for approximately one third of the total thoracohumeral elevation (Inman, Saunders, \& Abbot, 1944).

\section{Applications}

Several studies have been published on the relationship between scapular motion and thoracohumeral motion for clinical and diagnostic purposes. Vermeulen et al. (2002) reported on shoulder movement patterns in patients with a "frozen shoulder." They concluded that the frozen shoulder pattern was characterized by a relatively early laterorotation of the scapula. Ludewig and Cook (2002) have suggested that in patients with impingement syndrome, even small rotations might result in an important decrease of the subacromial space. In a comparable patient population, Hebert, Moffet, McFadyen, and Dionne (2002) did not find group differences between the healthy and the symptomatic shoulders, which might have been the result of a generally inappropriate neural strategy for these patients that affected both shoulders. Individual analyses revealed, however, less anterior tilting of the scapula in the symptomatic shoulder. Hebert et al. concluded that the lat-

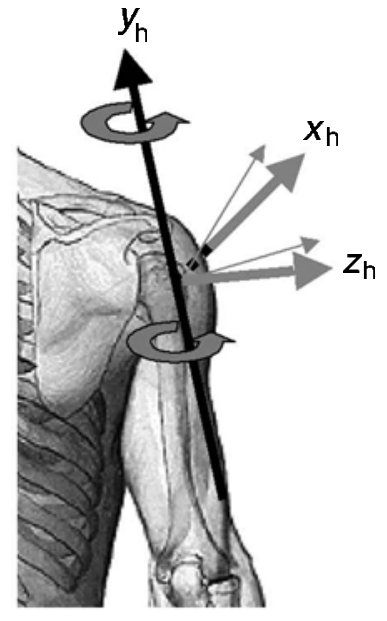

First rotation: Plane of elevation or pole angle

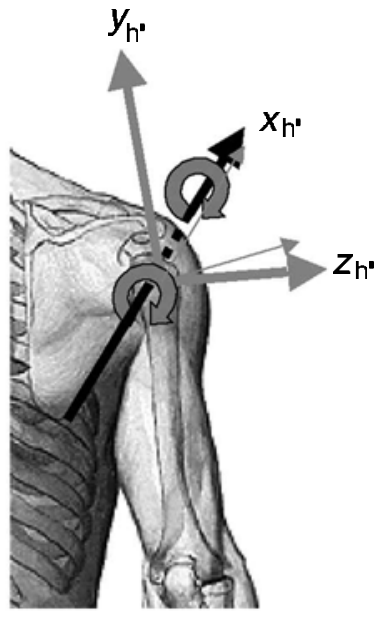

Second rotation: Elevation

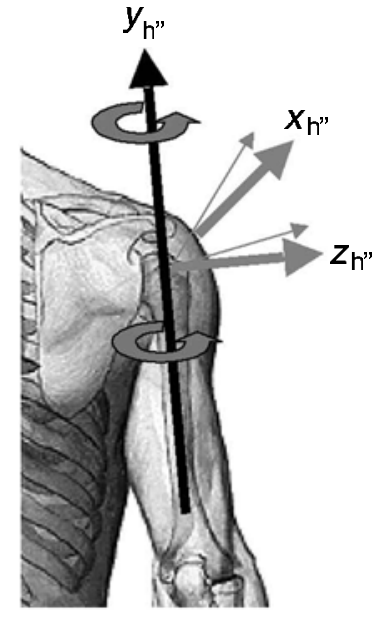

Third rotation: Axial rotation

Figure 4. Rotation order for the humerus, both the glenohumeral as well as the thoracohumeral rotations. The third rotation appears to be equal to the first but is, in fact, a rotation around the long axis of the humerus. The single quotes or double quotes indicate that the axes have been rotated, due to previous rotations. 
ter finding was evidence for focusing rehabilitation protocols toward a restoration of anterior tilting.

\section{Conclusions}

The protocol that is described here is an attempt to come to more standardized procedures for the measurement of arm-shoulder motions. A secondary aim of this article was to increase awareness of the limitations of the measurement of arm movements as thoracohumeral motions. Although previously practical reasons often prevented researchers from the analysis of arm movements in 3-D and as the complex of thoracoscapular and glenohumeral motions, the above protocol indicates that this does not have to apply. Both the technique and the procedures are now available and are, in fact, already in use today.

\section{REFERENCES}

An, K. N., Browne, A. O., Korinek, S., Tanaka, S., \& Morrey, B. F. (1991). Three-dimensional kinematics of glenohumeral elevation. Journal of Orthopaedic Research, 9, 143-149.

Anglin, C., \& Wyss, U. P. (2000). Arm motion and load analysis of sitto-stand, stand-to-sit, cane walking and lifting. Clinical Biomechanics, 15, 441-448.

Berme, N., Capozzo, A., \& Meglan, J. (1990). Rigid body mechanics as applied to human movement studies. In N. Berme \& A. Capozzo (Eds.), Biomechanics of human movement (pp. 89-107). Worthington, $\mathrm{OH}$ : Bertec Corporation.

DE GROOT, J. H. (1997). The variability of shoulder motions recorded by means of palpation. Clinical Biomechanics, 12, 461-472.

De Groot, J. H., \& Brand, R. (2001). A three-dimensional regression model of the shoulder rhythm. Clinical Biomechanics, 16, 735-743.

Doorenbosch, C. A. M., Mourits, A., Veeger, H. E. J., Harlaar, J., \& VAN DER HELM, F. C. T. (2001). Determination of functional rotation axes during elevation of the shoulder complex. Journal of Orthopaedic \& Sports Physical Therapy, 31, 133-137.

Hebert, L. J., Moffet, H., McFadyen, B. J., \& Dionne, C. E. (2002). Scapular behavior in shoulder impingement syndrome. Archives of Physical Medicine \& Rehabilitation, 83, 60-69.

Hebert, L. J., Moffet, H., McFadyen, B. J., \& St-Vincent, G. (2000). A method of measuring three-dimensional scapular attitudes using the Optotrak probing system. Clinical Biomechanics, 15, 1-8.

Högfors, C., Peterson, B., Sigholm, G., \& Herberts, P. (1991). Biomechanical model of the human shoulder joint: II. The shoulder rhythm. Journal of Biomechanics, 24, 699-709.

Inman, V. T., SAunders, J. B. D. M., \& Аввот, L. C. (1944). Observations on the function of the shoulder joint. Journal of Bone \& Joint Surgery, 26, 1-30.

Johnson, G. R., Stuart, P. R., \& Mitchell, S. (1993). A method for the measurement of three-dimensional scapular movement. Clinical Biomechanics, 8, 269-273.

Karduna, A. R., Ebaugh, D., Michener, L. A., \& McClure, P. W. (2002). The pattern of scapulohumeral motion: How stable is it? In Proceedings of the Fourth World Congress of Biomechanics, Calgary, 4-9 August 2002 [CD-ROM]

Karduna, A. R., McClure, P. W., \& Michener, L. A. (2000). Scapu- lar kinematics: Effects of altering the Euler angle sequence of rotations. Journal of Biomechanics, 33, 1063-1068.

Karduna, A. R., McClure, P. W., Michener, L. A., \& Sennett, B. (2001). Dynamic measurements of three-dimensional scapular kinematics: A validation study. Journal of Biomechanical Engineering, 123, 184-190.

Leroux, J. L., Micallef, J. P., Bonnel, F., \& Blotman, F. (1992). Rotation-abduction analysis in 10 normal and 20 pathological shoulders: Elite system application. Surgical \& RadiologicAnatomy, 14, 307-313.

Lu, T. W., LI, G. J., Kuo, M. Y., Chang, L. Y., \& Hsu, H. C. (2002). Measurement of three-dimensional kinematics of the glenohumeral joint during manual wheelchair propulsion using skeletal markers. In Proceedings of the Fourth World Congress of Biomechanics, Calgary, 4-9 August 2002 [CD-ROM].

Ludewig, P. M., \& CooK, T. A. (2002). Translations of the humerus in persons with shoulder impingement symptoms. Journal of Orthopaedic \& Sports Physical Therapy, 32, 248-259.

Meskers, C. G. M., van der Helm, F. C. T., Rozendaal, L. A., \& RozING, P. M. (1998). In vivo estimation of the glenohumeral joint rotation center from scapular bony landmarks by linear regression. Journal of Biomechanics, 31, 93-96.

Pascoal, A. G., van der Helm, F., Correia, P. P., \& Carita, I. (2000). Effects of different arm external loads on the scapulo-humeral rhythm. Clinical Biomechanics, 15, S21-S24.

Pearl, M. L., Harris, S. L., Lippitt, S. B., Sidles, J. A., Harryman, D. T., \& MATSEN, F. A. (1992). A system for describing positions of the humerus relative to the thorax and its use in the presentation of several functionally important arm positions. Journal of Elbow \& Shoulder Surgery, 1, 113-118.

Sharp, E. A., SuUr, J. L., \& Woledge, R. (2001). Measurements and interpretation of movements of the body surface during arm elevation. In E. K. J. Chadwick, H. E. J. Veeger, F. C. T. van der Helm, \& J. Nagels (Eds.) Proceedings of the Third Conference of the International Shoulder Group (pp. 52-55). Newcastle-upon-Tyne, U.K.: DUP Science.

StokdiJK, M., NAgels, J., \& RozIng, P. M. (2000). The glenohumeral joint rotation centre in vivo. Journal of Biomechanics, 33, 1629-1636.

van der Helm, F. C. T., Anglin, C., Chadwick, E., Karduna, A., Makhsous, M., McQuade, K., Nagels, J., van Roy, P., Veeger, H., \& WANG, X. (in press). ISB recommendation on definitions of joint co-ordinate system of various joints for the reporting of human joint motion: Pt. II. Shoulder. Journal of Biomechanics.

van der Helm, F. C. T., \& Pronk, G. M. (1995). Three-dimensional recording and description of motions of the shoulder mechanism. Journal of Biomechanical Engineering, 117, 27-40.

Vaughan, J., Rosenbaum, D. A., Harp, C. J., Loukopoulos, L. D., \& Engelbrecht, S. (1998). Finding final postures. Journal of Motor Behavior, 30, 273-284.

VEEGER, H. E. J. (2000). The position of the rotation center of the glenohumeral joint. Journal of Biomechanics, 33, 1711-1715.

Veeger, H. E. J., van der Helm, F. C. T., \& Rozendal, R. H. (1993). Orientation of the scapula in a simulated wheelchair push. Clinical Biomechanics, 8, 81-90.

Veeger, H. E. J., Yu, B., AN, K. N., \& Rozendal, R. H. (1997). Parameters for modeling the upper extremity. Journal of Biomechanics, 30, 647-652.

Vermeulen, H. M., Stokdijk, M., Eilers, P. H. C., Meskers, C. G. M., Rozing, P. M., \& Vlieland, T. (2002). Measurement of threedimensional shoulder movement patterns with an electromagnetic tracking device in patients with a frozen shoulder. Annals of the Rheumatic Diseases, 61, 115-120. 


\section{Definitions of Local Coordinate Systems (From van der Helm et al., in press)}

Each coordinate system is defined by three anatomical landmarks and an origin. The order in which the axes are defined is essential.

\section{Thorax Coordinate System: $x_{\mathrm{t}} y_{\mathrm{t}} z_{\mathrm{t}}$ \\ $o_{\mathrm{t}}: \quad$ The origin coincident with the IJ. \\ $y_{\mathrm{t}}$ : The line connecting the midpoint between PX and T8 and the midpoint between the IJ and $\mathrm{C}$, pointing upward. \\ $z_{\mathrm{t}}: \quad$ The line perpendicular to the plane formed by the IJ, C7, and the midpoint between PX and $\mathrm{T} 8$, pointing to the right. \\ $x_{\mathrm{t}}: \quad$ The common line perpendicular to the $x_{\mathrm{t}}$ - and the $y_{\mathrm{t}}$-axes, pointing forward.}

\section{Clavicle Coordinate System: $x_{\mathrm{c}} y_{\mathrm{c}} z_{\mathrm{c}}$}

$o_{\mathrm{c}}$ : The origin coincident with SC.

$z_{\mathrm{c}}$ : The line connecting AC and SC, pointing to AC.

$x_{\mathrm{c}}: \quad$ The line perpendicular to $z_{\mathrm{c}}$ and $y_{\mathrm{t}}(! !)$, pointing forward.

$y_{\mathrm{c}}: \quad$ The common line perpendicular to the $x_{\mathrm{c}}$ - and the $z_{\mathrm{c}}$-axis.

N.B. The $x_{\mathrm{c}}$-axis is defined with respect to the vertical axis of the thorax ( $y_{\mathrm{t}}$-axis), since only two bony landmarks can be discerned at the clavicle.

\section{Scapula Coordinate System: $x_{\mathrm{s}} y_{\mathrm{s}} z_{\mathrm{s}}$}

$o_{\mathrm{s}}: \quad$ The origin coincident with AA.

$z_{\mathrm{s}}: \quad$ The line connecting AA and TS, pointing to AA.

$x_{\mathrm{s}}: \quad$ The line perpendicular to the plane formed by AI, AA, and $x_{\mathrm{s}}$, pointing forward.

$y_{\mathrm{s}}: \quad$ The common line perpendicular to the $x_{\mathrm{s}}$ - and the $z_{\mathrm{s}}$-axis.

\section{Humerus (1st degree) Coordinate System: $x_{\mathrm{h} 1} y_{\mathrm{h} 1} z_{\mathrm{h} 1}$}

$o_{\mathrm{h} 1}: \quad$ The origin coincident with $\mathrm{GH}$.

$y_{\mathrm{h} 1}$ : The line connecting GH and the midpoint of EL and EM, pointing to GH.

$x_{\mathrm{h} 1}$ : The line perpendicular to the plane formed by EL, EM, and $y_{\mathrm{h} 1}$, pointing forward.

$z_{\mathrm{h} 1}$ : The common line perpendicular to the $y_{\mathrm{h} 1^{-}}$and the $z_{\mathrm{h} 1}$-axis.

\section{Humerus (2nd degree) Coordinate System: $x_{\mathrm{h} 2} y_{\mathrm{h} 2} z_{\mathrm{h} 2}$}

$o_{\mathrm{h} 2}: \quad$ The origin coincident with $\mathrm{GH}$.

$y_{\mathrm{h} 2}$ : The line connecting GH and the midpoint of EL and EM.

$z_{\mathrm{h} 2}$ : The line perpendicular to the plane formed by $y_{\mathrm{h} 2}$ and $y_{\mathrm{f}}$ (see below), pointing laterally.

$x_{\mathrm{h} 2}$ : The common line perpendicular to the $x_{\mathrm{h} 2}{ }^{-}$and the $y_{\mathrm{h} 2}$-axis.

N.B.1. The $z_{\mathrm{h} 2}$-axis depends on the position of the upper arm and the forearm, since it cannot be assured that it is equal to the joint rotation axis. Therefore, by definition, the $z_{\mathrm{h} 2}$-axis is taken with $90^{\circ}$ flexed elbow.

N.B.2. The 1 st and 2 nd degree definitions of the humerus coordinate system do not exactly agree, since the longitudinal axis of the forearm $\left(y_{\mathrm{f}}\right)$ is not necessarily perpendicular to the flexion/extension axis (see elbow definitions: Carrying angle), and the line EM-EL is not necessarily perpendicular to the longitudinal axis of the humerus.

\section{Forearm Coordinate System: $x_{\mathrm{f}} y_{\mathrm{f}} z_{\mathrm{f}}$}

$o_{\mathrm{f}}: \quad$ The origin coincident with the midpoint of RS and US.

$y_{\mathrm{f}}$ : The line connecting the midpoint of RS and US and the midpoint between EL and EM.

$z_{\mathrm{f}}$ : Perpendicular to the plane through US, RS, and the midpoint between EL and EM, pointing laterally.

$x_{\mathrm{f}}$ : $\quad$ Perpendicular to $y_{\mathrm{f}}$ and $z_{\mathrm{f}}$, pointing forward. 